\title{
A heat-shock 20 protein isolated from watermelon (CIHSP22.8) negatively regulates the response of Arabidopsis to salt stress via multiple signaling pathways
}

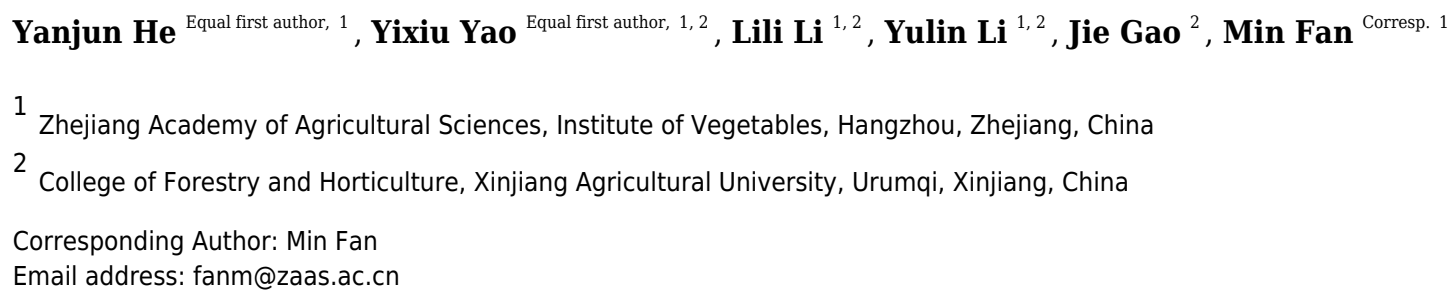

Heat-shock protein 20s (HSP20s) were initially shown to play a role during heat shock stress; however, recent data indicated that HSP20 proteins are also involved in abiotic stress in plants. Watermelon is known to be vulnerable to various stressors; however, HSP20 proteins have yet to be investigated and characterized in the watermelon . In a previous study, we identified a negative regulator of salt stress response from watermelon: CIHSP22.8, a member of the HSP20 family. Quantitative real-time PCR (qRT-PCR) and promoter:: $\beta$-glucuronidase (GUS) analysis revealed that CIHSP22.8 was expressed widely in a range of different tissues from the watermelon, but particularly in the roots of 7-dayold seedlings and flowers. Furthermore, qRT-PCR and GUS staining showed that the expression of CIHSP22.8 was significantly repressed by exogenous abscisic acid (ABA) and salt stress. The over-expression of CIHSP22.8 in Arabidopsis lines resulted in hypersensitivity to $A B A$ and reduced tolerance to salt stress. Furthermore, the expression patterns of key regulators associated with ABA-dependent and independent pathways, and other stress-responsive signaling pathways, were also repressed in transgenic lines that over-expressed CIHSP22.8. These results indicated that CIHSP22.8 is a negative regulator in plant response to salt stress and occurs via ABA-dependent and independent, and other stress-responsive signaling pathways. 
1 A heat-shock 20 protein isolated from watermelon

2 (CIHSP22.8) negatively regulates the response of

3 Arabidopsis to salt stress via multiple signaling

4 pathways

5 Yanjun $\mathrm{He}^{1 \#}$, Yixiu Yao ${ }^{1,2 \#}$, Lili $\mathrm{Li}^{1,2}$, Yulin $\mathrm{Li}^{1,2}$, Jie Gao ${ }^{2}$, and Min Fan ${ }^{1 *}$

$6{ }^{1}$ Zhejiang Academy of Agricultural Sciences, Institute of Vegetables, Hangzhou 310021, China;

$7{ }^{2}$ College of Forestry and Horticulture, Xinjiang Agricultural University, Urumqi, Xinjiang,

8 China

$9 \quad$ \# These authors contributed equally to this work.

$10 *$ Correspondence:

11 Min Fan

12 Zhejiang Academy of Agricultural Sciences, Institute of Vegetables, Hangzhou 310021, China;

13 fanm@zaas.ac.cn;

14

15

16

17

18

19

20

21

22

23

24

25

26

27

28

29

30

31

32

33

34 35

\section{Abstract}

Heat-shock protein 20s (HSP20s) were initially shown to play a role during heat shock stress; however, recent data indicated that HSP20 proteins are also involved in abiotic stress in plants. Watermelon is known to be vulnerable to various stressors; however, HSP20 proteins have yet to be investigated and characterized in the watermelon. In a previous study, we identified a negative regulator of salt stress response from watermelon: ClHSP22.8, a member of the HSP20 family. Quantitative real-time PCR (qRT-PCR) and promoter:: $\beta$-glucuronidase (GUS) analysis revealed that $C l H S P 22.8$ was expressed widely in a range of different tissues from the watermelon, but particularly in the roots of 7-day-old seedlings and flowers. Furthermore, qRT-PCR and GUS staining showed that the expression of ClHSP22.8 was significantly repressed by exogenous abscisic acid (ABA) and salt stress. The over-expression of ClHSP22.8 in Arabidopsis lines resulted in hypersensitivity to $\mathrm{ABA}$ and reduced tolerance to salt stress. Furthermore, the expression patterns of key regulators associated with ABA-dependent and independent pathways, and other stress-responsive signaling pathways, were also repressed in transgenic lines that overexpressed ClHSP22.8. These results indicated that ClHSP22.8 is a negative regulator in plant response to salt stress and occurs via ABA-dependent and independent, and other stress-responsive signaling pathways.

Keywords: ClHSP22.8; Watermelon (Citrullus lanatus); Salt stress; ABA; Arabidopsis (Arabidopsis thaliana)

\section{Introduction}

Heat-shock proteins (HSPs) act as molecular chaperones and are found in all species of plants. HSPs help to protect their target proteins from denaturation, misfolding, and aggregation, during 
36

37

38

39

40

41

42

43

44

45

46

47

48

49

50

51

52

53

54

55

56

57

58

59

60

61

62

63

64

65

66

67

68

69

70

71

72

73

74

75

times of stress ${ }^{[1,2]}$. Previous research has shown that HSPs can be classified into six groups based on molecular weight: HSP100, HSP90, HSP70, HSP60, HSP20 (or small heat-shock protein), and ubiquitin ${ }^{[2,3]}$. HSP20 is now known to be the largest and best studied family of the HSP families [4]. Numerous studies have revealed specific roles for plant HSP20 proteins in a range of abiotic stress responses. For example, transgenic plants that over-expressed HSP2Os was shown to exhibit enhanced tolerance to heat, including Arabidopsis, rice, wheat, maize, and Chenopodium ${ }^{[3,5-9]}$. Another study showed that the over-expression of OSHSP20s in rice led to enhanced tolerance to stress caused by ultraviolet-B radiation, salt, drought, and dehydration [6,10,11]. Other research studies have shown that the over-expression of OSHSP17.0 or OsHSP23.7 led to an improvement in the drought and salt tolerance of rice and that this involved a reduction of membrane damage and increased expression of protective molecules [10]. Research has shown that OSHSP18.2 is implicated in seed vigor and longevity and can improve germination and the successful creation of seedlings under abiotic stress ${ }^{[11]}$. Promoter analyses further revealed that the over-expression of TaHSP26 in wheat could be induced by heat, cold, salt, and drought ${ }^{[12]}$. Furthermore, the overexpression of TaHSP23.9 in wheat led to an enhancement in the tolerance to heat and salt stresses [13]. Another study, involving the ectopic expression of LimHSP16.45 led to an increase in the activities of superoxide dismutase (SOD) and catalase (CAT), thus improving the vigor of seed germination in Arabidopsis under salt stress ${ }^{[14]}$. The over-expression of ZmHsp 16.9 in tobacco led to an enhancement in the activities of peroxidase (POD), CAT, and SOD, and an increase in oxidative stress tolerance ${ }^{[5]}$. HSP20s have been found to regulate the plant response to salt stress via abscisic acid (ABA) signaling pathways. In Capsicum annuum, CaHsp22.5 was shown to modulate plant ABA signaling and participate in response to salt stress ${ }^{[15]}$. Other research, carried out in creeping bentgrass, showed that AsHSP17 and AsHSP26.8a mediate ABA-dependent and independent and other stress signaling pathways to negatively regulate plant responses to salt stress [16,17].

Watermelon (Citrullus lanatus L.) is an economically important cucurbit crop that is cultivated across the world. However, it is vulnerable to a variety of adverse environmental conditions ${ }^{[18]}$. As one of the most important stressors, salinity stress can lead to serious limitations in the yield and quality of watermelon ${ }^{[19]}$. HSP20s are the most abundant HSP sub-type in plants and are known to play important functions in a variety of stress responses ${ }^{[5,10,13-17]}$. However, we know very little about the specific role of watermelon $H S P 20 s$ with regards to abiotic stress tolerance.

In a previous study, we identified the HSP20 gene family in watermelon and analyzed their expression patterns in response to different stresses ${ }^{[20]}$. In the present study, we characterized an HSP20 gene (ClHSP22.8) from the watermelon. Quantitative real-time PCR (qRT-PCR) and promoter:: $\beta$-glucuronidase (GUS) assays showed that the expression of ClHSP22.8 was repressed by exogenous ABA and salt treatment. Next, we successfully constructed Arabidopsis lines that over-expressed ClHSP22.8 and used these lines to investigate their sensitivity to ABA and salt tolerance. We analyzed the expression profiles of genes related to $\mathrm{ABA}$ and the stress response of plants that over-expressed ClHSP22.8 under salt treatment. Functional studies of ClHSP22.8 will not only provide a better understanding of the specific roles of HSP20s in the adaption of 
watermelon to salt stress but may also provide insight into the potential signaling processes in response to stressful conditions.

\section{Materials \& Methods}

\section{Identification of CIHSP22.8 and bioinformatics analysis}

First, we downloaded the amino acid sequence of ClHSP22.8 from the Cucurbit Genomics Database (accession number: Cla017945). We then used ExPASy (http://web.expasy.org/ computepi/) to calculate the molecular weight (MW) and isoelectric point (pI). Conserved domains in the ClHSP22.8 protein were confirmed using the SMART database (http://smart.emblheidelberg. de/). Next, we downloaded the protein sequences of various ClHSP22.8 orthologs in Arabidopsis, rice, tomato, soybean, switchgrass, and cucumber, from Phytozome (http://phytozome.jgi.doe.gov/ pz/portal.html). Then, we performed phylogenetic analysis, based on the full-length protein sequences, using the MEGA 5.0 program and the neighbor-joining (NJ) method with 1000 bootstrap replicates ${ }^{[21]}$. Multiple sequence alignment of the predicted peptide sequences of the conserved-crystallin (ACD) domain was then carried out using Clustal X version 1.81 with default parameters ${ }^{[22]}$. The ClHSP22.8 promoter sequence was also downloaded from the Cucurbit Genomics Database and subsequently analyzed via PlantCARE (http://bioinformatics.psb.ugent. be/webtools/plantcare/ html/).

\section{Plant materials and growth conditions}

We used the watermelon advanced inbred line 'JJZ-M' for all expression analyses. These plants were grown in a growth chamber in temperature-controlled greenhouses under day/night temperatures of $28 / 22 \pm 1^{\circ} \mathrm{C}$, a light intensity of $200 \mu \mathrm{mol} \mathrm{m} \mathrm{m}^{-2} \mathrm{~s}^{-1}$, and a $16-\mathrm{h} \mathrm{light} / 8-\mathrm{h}$ dark photoperiod. Three-week-old watermelon seedlings were used for treatments involving exogenous ABA and salt stress treatments; these treatments involved the seedlings being sprayed with 100 $\mu \mathrm{M}$ of $\mathrm{ABA}$ and $200 \mathrm{mM}$ of $\mathrm{NaCl}$, respectively ${ }^{[20]}$. The second true leaf on each plant was sampled at time 0 (control), and then again at 1, 4, and $12 \mathrm{~h}$ after treatment. Arabidopsis thaliana ecotype 'Columbia' (wild type, WT) plants were used for the construction of transgenic plants. These plants were kept at $24 / 22^{\circ} \mathrm{C}$ (16 h-day/8 h-night) with $65 \%$ relative humidity to yield transgenic lines. Both transgenic and WT plants were cultivated under the same growth conditions.

\section{Quantitative real-time PCR}

Total RNA was isolated from samples of both watermelon and Arabidopsis. Reverse transcription was then performed using the PrimeScript RT reagent kit (Takara, China) in accordance with the manufacturer's instructions; for each sample, approximately $1 \mu \mathrm{g}$ of total RNA was reverse transcribed into cDNA. Quantitative real-time PCR (qRT-PCR) reactions were performed on a CFX96 Real Time PCR System (Bio-Rad, USA) using the following cycle conditions: $30 \mathrm{~s}$ at $95^{\circ} \mathrm{C}$; followed by 40 cycles of $5 \mathrm{~s}$ at $95^{\circ} \mathrm{C}$, and $45 \mathrm{~s}$ at $55^{\circ} \mathrm{C}$; this was followed by 1 cycle of 1 
$111 \mathrm{~min}$ at $95^{\circ} \mathrm{C}, 30 \mathrm{~s}$ at $50^{\circ} \mathrm{C}$ and $30 \mathrm{~s}$ at $95^{\circ} \mathrm{C}$. Two biological and three technical replicates were 112 carried out for each sample; these reactions involved a reaction volume of $15 \mu \mathrm{L}$ and the SYBR 113 Premix Ex Taq kit (Toyobo, Japan). We used the watermelon $\beta$-actin gene and the Arabidopsis 114 ACTIN2 gene as reference sequences for primer design (Table S1) and relative gene expression 115 was calculated using the $2^{-\triangle \triangle \mathrm{Ct}}$ method.

116 Promoter assay by GUS histochemical staining

117 In order to investigate the tissue-specific expression of ClHSP22.8, we amplified a $1601 \mathrm{bp}$ 118 upstream promoter sequence using specific primer pairs (Table S1). We then ligated this fragment 119 with the pBI101 plasmid vector that could be subsequently transformed into Arabidopsis. The 120 reporter construct containing the GUS reporter gene driven by the ClHSP22.8 promoter region 121 was named ProClHSP22.8::GUS (Figure S1). Transgenic Arabidopsis seedlings were obtained 122 using the floral dip method [23]. Transgenic Arabidopsis seeds were screened using $100 \mathrm{mg} \mathrm{L}^{-1}$ 123 kanamycin (KanR). Positive transgenic plants were identified by GUS histochemical staining. T3 124 transgenic lines were also screened and harvested for further phenotypic observation.

125 The 7-day-old Arabidopsis transgenic seedlings created from the T3-generation grown on 1/2 MS 126 medium were transferred to $1 / 2 \mathrm{MS}$ medium with and without $100 \mu \mathrm{M}$ of ABA and $200 \mathrm{mM}$ of $127 \mathrm{NaCl}$. After $24 \mathrm{~h}$, the transgenic seedlings were GUS stained using a GUS Histochemical Staining 128 Kit (O'BioLab, Beijing, China) in accordance with the manufacturer's guidelines. After removing 129 chlorophyll with $70 \%$ ethanol, we analyzed the seedlings and acquired typical digital images using 130 a stereomicroscope (STEMI SV11, Zeiss, Jena, Germany).

\section{Plasmid construction and generation of transgenic plants}

132 The coding sequence (CDS) of ClHSP22.8 was amplified using a pair of specific primers: 133 ClHSP22.8-F and ClHSP22.8-R (Table S1). The amplicons produced by PCR were subsequently 134 digested and ligated into the pBI121 vector (Figure S1). Subsequently, the pBI121135 p35S::ClHSP22.8 vector was transformed into Arabidopsis using the floral dip method. 136 Transgenic Arabidopsis lines were confirmed by PCR using two specific primers: HSP22.8-S and 137 GUS-A (Figure S2). The progenies of these plants were screened using $100 \mathrm{mg} \mathrm{L}^{-1} \mathrm{KanR}$, as 138 described earlier. As a result, four independent homozygous transgenic lines were created; we 139 named these OE22.8-1, OE22.8-2, OE22.8-3, and OE22.8-4. T3 transgenic lines were screened 140 and harvested for further phenotypic observation.

ABA and salt tolerance in transgenic Arabidopsis lines

142 The T3-generation transgenic lines (OE22.8-1 to OE22.8-4) were grown with WT seedlings on $1431 / 2 \mathrm{MS}$ medium with or without $100 \mu \mathrm{M}$ of ABA and $200 \mathrm{mM}$ of $\mathrm{NaCl}$, respectively. After 7 days, 144 we photographed these plants so that we had a record of their relative phenotypes. The growth 145 status and root length of two-week-old plants were measured, and samples were taken for qRT- 
146 PCR. Three independent biological replicates were analyzed; each replicate involved over 30 147 seedlings.

\section{Statistical analysis}

149 Data were analyzed by a two-tailed Student's $t$-test or by one-way analysis of variance (ANOVA)

150

151

152

153

154

155

156

157

158

159

160

161

162

163

164

165

166

167

168

169

170

171

172

173

174

175

176

177 Response patterns of watermelon CIHSP22.8 to ABA and salt stress

using SPSS version 18.0 (IBM, Chicago, IL, United States). ${ }^{*} \mathrm{P}<0.05$ and $* * \mathrm{P}<0.01$ were considered to be significant and highly significant, respectively.

\section{Results}

\section{Isolation and bioinformatics analysis of CIHSP22.8}

The length of the full-length coding sequence (CDS) for the ClHSP22.8 gene was $582 \mathrm{bp}$ and coded for a protein containing 193 amino acids. The isoelectric point (pI) of the protein was 7.76, and the molecular weight (MW) was $22.85 \mathrm{kDa}$ (Table S1). ClHSP22.8 on Chr 10 was mapped to a segmentally duplicated region that was shared with ClHSP16, ClHSP17.6C, and ClHSP17.6D, respectively (Table S1). The ClHSP22.8 protein shared a conserved $\alpha$-crystallin ACD/HSP20 domain (thus showing conservation of region I and II) with its orthologs (Figure 1A). Phylogenetic analysis further showed that ClHSP22.8 and CsHSP23.7 in the cucumber formed a separate branch that was distant from a range of other orthologs from Arabidopsis, rice, tomato, soybean, and switchgrass (Figure 1B).

\section{Spatial and temporal expression of CIHSP22.8 in watermelon}

Next, we determined the spatial and temporal expression profiles of ClHSP22.8 via qRT-PCR. Data indicated that $C l H S P 22.8$ was widely expressed across a range of different tissues in the watermelon and showed the lowest level in the fruit; higher levels of expression were observed in female flowers and roots (51.37- and 26.63-fold compared to that in the fruit) (Figure 2A). In order to further characterize the tissue-specific expression of the ClHSP22.8 gene, we amplified a 1601 bp fragment from a region that was upstream of the ATG start codon in the ClHSP22.8 gene. We then transfected this fragment into Arabidopsis in order to drive $\beta$-glucuronidase (GUS) gene expression (Figure S1; Figure 2B-G). Results from GUS histochemical staining indicated that the GUS protein was expressed at the highest levels in roots; the next highest level of expression was seen in the cotyledons, especially in the leaf vein. Signals were also detected in the vascular tissue of hypocotyls in 7-day-old Arabidopsis seedlings (Figure 2B). In adult Arabidopsis plants, the GUS signal was expressed at the highest levels in flowers, followed by leaves. Only weak signals were detected in the stem, silique, and roots (Figure 2C-G). 
178 The expression patterns of ClHSP22.8 in response to ABA and salt stress were detected by qRT179 PCR in watermelon leaves at four different timepoints $(0,1,4$, and $12 \mathrm{~h})$. Results indicated that 180 ClHSP22.8 expression was obviously downregulated by 0.52 - and 0.14 -fold at 4 and $12 \mathrm{~h}$ after 181 ABA treatment, respectively (Figure 3A). Similarly, the expression of ClHSP22.8 was 182 significantly reduced by salt stress and reached a minimum at $4 \mathrm{~h}(0.16$-fold $)$, although the level 183 of reduction appears to weaken at $12 \mathrm{~h}(0.61$-fold) (Figure 3B).

184 Cis-acting elements of the ClHSP22.8 promoter sequence were subsequently analyzed via 185 PlantCARE website. Two ABA responsiveness elements (ABREs), one anaerobic responsiveness 186 element (ARE), and two MeJA-responsiveness elements (TGACG-motifs) were identified (Figure 3C); Two ABRE elements were located at -501 and $-379 \mathrm{bp}$; the ARE element was located at 188 $1524 \mathrm{bp}$; and two MeJA-responsiveness elements were located at -1477 and -381 bp. We were not able to detect an HSE motif in the promoter.

190

191

192 ProClHSP22.8::GUS analysis showed that there were no significant differences in terms of GUS staining in roots of 7-day-old seedlings in response to ABA and salt treatment at $24 \mathrm{~h}$ when compared with untreated seedlings, although there was a significant reduction in the leaves (Figure 3D-F).

\section{Overexpression of CIHSP22.8 conferred ABA sensitivity to Arabidopsis}

195

196

197

198

199

200

201

202

203

204

205

206

207

208

209

210

211

212

213

We constructed Arabidopsis lines that over-expressed ClHSP22.8 and screened these lines by both PCR and qRT-PCR (Figure S2; Figure 4). Compared to the WT as a control, we found that the ClHSP22.8 gene was significantly over-expressed by 8.60-, 20.32-, 16.38-, and 27.20-fold in the OE22.8-1, OE22.8-2, OE22.8-3, and OE22.8-4 lines, respectively (Figure 4A). To determine the ABA sensitivity of the lines that over-expressed ClHSP22.8, we grew the OE22.8 lines, along with the WT plants, on $1 / 2 \mathrm{MS}$ medium with $100 \mu \mathrm{M}$ of ABA. After 7 days, we found that the root growth of the WT plants had decreased by $29.61 \%$ in response to exogenous ABA treatment when compared with untreated WT seedlings (defined as mock). However, we found that root growth in the OE22.8-1, OE22.8-2, OE22.8-3, and OE22.8-4 seedlings was seriously repressed, by $36.88 \%$ to $61.08 \%$, when compared to the WT plants (Figure 4B-E). This data indicated that plants that over-expressed ClHSP22.8 were more sensitive to ABA treatment.

\section{Overexpression of CIHSP22.8 reduced tolerance to salt in transgenic Arabidopsis}

In order to further investigate the specific roles of ClHSP22.8 in response to salt stress, we subjected 7-day-old seedlings that over-expressed ClHSP22.8 (OE22.8-1, OE22.8-2, and OE22.84 lines) and WT plants to $200 \mathrm{mM} \mathrm{NaCl}$. After 7 days, the seedlings that over-expressed ClHSP22.8 showed more chlorosis and stunted phenotypes; their primary root lengths were also more significantly reduced than the WT plants (Figure 4). Compared with mock seedlings, we found that the root length in WT plants after salt treatment was reduced by $8.87 \%$ in response to salt treatment. In contrast, the OE22.8 lines showed a more serious reduction in root length 
214 (19.35\% to 50.43\%). This data indicated that ClHSP22.8 negatively regulates plant salinity stress 215 response.

216 Some ABA- and stress-related genes were repressed by over-expression of 217 CIHSP22.8 under salt stress

218 Finally, we used qRT-PCR to investigate the expression profiles of several representative genes 219 that are involved in ABA biosynthesis and signaling and stress-responsive transcription factors 220 (TFs), including Arabidopsis 9-cis epoxycarotenoid dioxygenase 3 (AtNCED3), ABA insensitive 2214 (AtABI4), ethylene response factor 05 (AtERF05), Arabidopsis dehydration-responsive element222 binding protein 1B $(A t D R E B 1 B)$, zinc finger protein (AtZAT7), and myb domain protein 44 223 (AtMYB44). In the WT plants, we found that AtNCED3, AtABI4, and AtERF05 were repressed by 224 0.28- to 0.57-fold, while AtDREB1B, AtMYB44, and AtZAT7, were induced by 2.22- to 4.35-fold 225 after salt treatment (Figure S3). These results indicate that these six genes are salt-responsive 226 genes. Furthermore, the expressions of these six genes were significantly repressed in the OE22.82272 (from 0.47- to 0.58-fold) and OE22.8-4 (from 0.32- to 0.46-fold) lines compared to that in the 228 WT plants (Figure 5). This data indicated that the overexpression of ClHSP22.8 could repress the 229 expression of these stress-responsive genes in Arabidopsis.

230 In order to confirm whether the reduction/elevation in expression was more pronounced in the 231 OE22.8 lines when treated with $200 \mathrm{mM} \mathrm{NaCl}$, we normalized the changes observed in WT plants 232 and then checked if the OE22.8 lines showed statistically significant differences in terms of gene 233 expression (Figure 6). We observed a significant reduction in the expression of AtNCED3, AtABI4, 234 and $A t D R E B 1 B$ in the OE22.8-2 and OE22.8-4 lines after salt treatment. The expression levels of 235 AtERF05 and AtMYB44 were obviously repressed, but only in the OE22.8-4 line. There were no 236 significant differences in the expression levels of AtZAT7 in the OE22.8 lines after salt treatment. 237 These results indicate that all of the detected genes, except AtZAT7, might represent target genes 238 for ClHSP22.8-mediated repression in response to salt stress.

\section{Discussion}

240

241

242

243

244

245

246

247

248

249

250

Salinity is an important environmental stress factor and can have a severe effect on the growth and development of plants. Consequently, salt stress is a growing problem for global agricultural production ${ }^{[24]}$. Watermelon (Citrullus lanatus) is a salt-sensitive crop and may help us to engineer more salt-tolerant varieties so that we can investigate the core salt-tolerance mechanisms in this fruit ${ }^{[19]}$. As the largest family, and most well studied HSP, the HSP20 family of proteins is ATPindependent and generally assemble into large oligomers that can protect other proteins from denaturation and aggregation ${ }^{[1,2]}$. An increasing body of evidence has shown that HSP2Os regulate the responses of plants to environmental changes and thus allow plants to survive adverse conditions ${ }^{[5-17]}$. In a previous study, we identified HSP20 genes within the genome of watermelon and found that HSP20s in plants can be divided into 18 subfamilies [20]. The largest group is the nucleocytoplasmic (C)-located HSP20s; this group features 13 subfamilies that exhibit functional 
251

252

253

254

255

256

257

258

259

260

261

262

263

264

265

266

267

268

269

270

271

272

273

274

275

276

277

278

279

280

281

282

283

284

285

286

287

288

289

290

redundancy and divergence ${ }^{[20,25,26]}$. As a member of the CIX subfamily, ClHSP22.8 has a ACD domain, agglomerates into granules in the cytoplasm, and existes as larger oligomers in vivo as expected ${ }^{[20]}$. Gene duplication events are major sources of new gene functions ${ }^{[27]}$. ClHSP22.8 was duplicated with ClHSP17.6C, ClHSP17.6D, and ClHSP16, from the CI subfamily, but it does not have a close relationship with the duplicates, or homologs in other species, except for CsHSP23.7 in cucumber (Figure 1B, Table S1) ${ }^{[20]}$. Notably, we found ClHSP22.8 could not be induced by heat but was significantly repressed by salt stress (Figure 3B), which was different from a typical HSP20 that exhibited rapid and significant upregulation under heat stress ${ }^{[1,2,20]}$. Therefore, we consider that ClHSP22.8 probably evolved new functionality in stress responses following the gene duplication event ${ }^{[27]}$.

In order to verify the precise functional role of ClHSP22.8 in salt stress response, we created lines of Arabidopsis that over-expressed ClHSP22.8 and found that these overexpression lines exhibited shorter roots and more yellow leaves under salt stress. Thus, these results indicate that ClHSP22.8 negatively regulates the salinity tolerance of Arabidopsis (Figure 4). In this study, we used qRTPCR and promoter::GUS analysis analysis and found that ClHSP22.8 was clearly repressed by salt treatment (Figure 3B). The transcript abundance of ClHSP22.8 in response to salt stress reached the lowest level in the first 4 hours. The response pattern of ClHSP22.8 that rapidly and sharply responded to salt stress in a short time and then had slight variations, was similar to quite a few HSP20s such as TaHSP23.9 [13], OsHSP20 [25], AsHSP17 [16] and so on. The response pattern of $H S P 20 s$ probably is a kind of mechanism for plants rapidly adapt to salt stress.

$H S P 20 s$ are known to modulate the multiple signaling pathway so as to regulate the plant's response to salt stress. Most HSP20s have been reported to play positive roles in regulating plant tolerance to salt, including maize ZmHsp16.9 [5], rice OsHSP17.0 and OsHSP23.7 [10], wheat

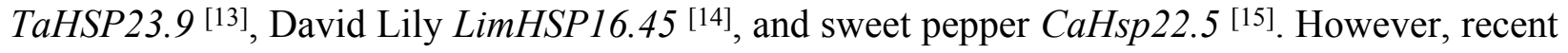
work, a few studies about the negative effect of HSP20s on plant response to salt stress have been reported. The over-expression of AsHSP17 or AsHSP26.8 in plants led to the direct repression of the vast majority of stress-responsive genes involved in plant photosynthesis, ABA-dependent and ABA-independent pathways, and some other stress response pathways, thus led to reduced levels of salt tolerance ${ }^{[16,17]}$, but the negative regulation mechanisms of HSP20s in salt stress responses remain to be fully unraveled. Similar to AsHSP17 and AsHSP26.8 [16,17], ClHSP22.8 also plays negative roles in terms of salt response involved in ABA signaling pathway. ABA responsiveness element (ABRE) and anaerobic response element (ARE) cis-elements can be recognized by $\mathrm{AREB} / \mathrm{ABF}$ and MYB transcription factors, respectively ${ }^{[28,29]}$. The two cis-elements are necessary for ABA- and anaerobic-responsive gene expression ${ }^{[28,29]}$. In the present study, two ABRE and one ARE elements were identified in the ClHSP22.8 promoter (Figure 3C). And ClHSP22.8 was significantly repressed by the exogenous ABA via qRT-PCR and promoter:: $G U S$ analysis (Figure $3 \mathrm{~A}, \mathrm{D}$, and E). Above results indicate that ClHSP22.8 respond to salinity stress in a negative manner and that this response involved the ABA mediated signaling pathway.

To further illustrate the regulatory mechanism of ClHSP22.8 under salt stress, we used qRT-PCR to determine the expression patterns of six ABA- and stress-related genes in response to salt stress; 
291 results demonstrated that all of these detected genes could obviously respond to salt stress which 292 AtNCED3, AtABI4, and AtERF05 were obviously repressed while AtDREB1B, AtMYB44, and 293 AtZAT7 were induced by salt stress (Figure S3). Meanwhile, studies found that over 90\% 294 differentially expressed genes (DEGs) in AsHSP17 and AsHSP26.8 overexpressed lines were 295 down-regulated ${ }^{[16,17]}$. Similarly, all of the detected genes in this study were significantly repressed 296 by overexpression of ClHSP22.8. These results indicate all of the six detected genes are salt297 responsive genes and repressed by ClHSP22.8.

298 ABA-dependent pathways is an important mechanism in adaptation to salt stress and affacts plant 299 salt stress response and tolerance [30,31]. ABA biosynthesis and signalling and some stress300 responsive transcription factors involved in ABA signalling were key regulators in ABA301 dependent pathway. Among the detected genes in this study, AtNCED3 encodes a rate-limiting 302 enzyme that plays a role in ABA biosynthesis and ABI4 is a key regulator of the ABA-dependent 303 pathway ${ }^{[30,31]}$. Their expression were significantly repressed in OE22.8 lines after normalized the 304 changes observed in WT plants (Figure 6). So the over-expression of ClHSP22.8 probably resulted 305 in lower levels of ABA and enhanced ABA sensitivity by repressing the expression of AtNCED3 306 and $A B I 4$ transcripts in response to salt stress. Besides, some $M Y B$ and $Z A T$ transcription factors 307 have been implicated in the plant response to abiotic stress and ABA sensitivity ${ }^{[32-37]}$. AtMYB44 308 was involved in ABA-dependent signaling pathways that regulate stress adaption and confer plant 309 tolerance to salt stress [33,36,37]. And the constitutive expression of AtZAT7 suppressed growth and 310 enhanced salt tolerance in transgenic Arabidopsis plants ${ }^{[32]}$. AtMYB44 and AtZAT7 have both been 311 shown to be regulated by HSP20s in response to salt stress ${ }^{[16,17]}$. In this study, we found that the 312 expression of AtMYB44 and AtZAT7 can be obviously induced by salt in WT plants but the 313 reduction of AtMYB44 is more pronounced in the OE22.8-4 plants (Figure S3, Figure 6). These 314 results indicated that $C l H S P 22.8$ participates in the response to salt stress via an ABA-dependent 315 pathway.

316 Our data suggested ClHSP22.8 also modulated the response to salt stress via an ABA-independent 317 signaling pathway. Previous work has shown that AtDERB1B and AtERF05 participate in ABA318 independent pathway [17,31]; and we found AtDERB1B and AtERF05 were both ClHSP22.8319 regulated salt responsive genes. The expression of $A t D E R B 1 B$ were obviously induced while 320 AtERF05 were repressed by salt stress in WT plants (Figure S3). And they were both significantly 321 repressed in OE22.8 lines (Figure 5). In further, the two genes were significantly repressed by salt 322 stress in OE22.8 lines after normalizing the effects shown in WT plants (Figure 6).

323 Collectively, these results imply that, as a negative regulator of salt stress, ClHSP22.8 may be 324 repressed to an appropriate level in protecting plants from salt stress. However, when $C l H S P 22.8$ 325 was overexpressed in Arabidopsis, some genes involved in ABA-dependent (AtNCED3 and ABI4) 326 and ABA-independent (AtDERB1B and AtERF05) signaling pathways, and stress-responsive TF 327 (AtMYB44) in ABA signalling were repressed, and then the salt stress response regulatory network 328 was negatively impacted as outlined in Figure 7. Our data provides further understanding of the 329 specific roles of HSP20s in the watermelon in terms of the response to abiotic stress.

\section{Conclusions}


331

332

333

334

335

336

337

338

339

340

341

342

343

344

345

346

347

348

349

350

351

352

353

354

355

356

357

358

359

360

361

362

363

364

365

366

367

368

369

370

371

372

In summary, we identified an $H S P 20$ gene, ClHSP22.8, and demonstrated that this gene plays important roles in the salt response. Analyses involving both qRT-PCR and promoter::GUS analysis indicated that the expression of ClHSP22.8 in Arabidopsis could be repressed by exogenous ABA and salt stress. Furthermore, the over-expression of ClHSP22.8 repressed some genes that are known to be involved in ABA-dependent and independent signaling pathways, and other stress-responsive pathways, thus leading to an enhanced level of plant sensitivity to ABA and a reduced tolerance to salt stress. Our study provides a better understanding of the specific roles of $H S P 20 \mathrm{~s}$ in watermelon with regards to the abiotic stress response and suggests that ClHSP22.8 may be a valuable gene to consider when cultivating watermelons.

Funding: This research was funded by the Natural Science Foundation of Zhejiang Province (LQ18C150003), and National Natural Science Foundation of China (31772332).

Conflicts of Interest: The authors declare no conflict of interest.

\section{References}

1. Papsdorf, K.; Richter, K. Protein folding, misfolding and quality control: the role of molecular chaperones. Essays Biochem. 2014, 56, 53-68.

2. Asea, A.A.A.; Kaur, P.; Calderwood, S.K. Heat Shock Proteins and Plants. Springer International Publishing:Cham, Switzerland. 2016.

3. Sun. Y.; MacRae, T.H. Small heat shock proteins: molecular structure and chaperone function. Cell Mol Life Sci. 2005, 62, 2460-2476.

4. Basha, E.; O’Neill, H.; Vierling, E. Small heat shock proteins and a-crystallins: dynamic proteins with flexible functions. Trends Biochem Sci. 2012, 37, 106.

5. Sun, L.P.; Liu, Y.; Kong, X.P.; Zhang, D.; Pan, J.W.; Zhou, Y.; Wang, L.; Li, D.Q.; Yang, X.H. ZmHSP 16.9, a cytosolic class I small heat shock protein in maize (Zea mays), confers heat tolerance in transgenic tobacco. Plant Cell Rep. 2012, 31, 1473-1484.

6. Murakami, T.; Matsuba, S.; Funatsuki, H.; Kawaguchi, K.; Saruyama, H.; Tanida, M.; Sato, Y. Over-expression of a small heat shock protein, sHSP17.7, confers both heat tolerance and UV-B resistance to rice plants. Mol Breed. 2004, 13, 165-175.

7. Charng, Y.Y.; Liu, H.C.; Liu, N.Y.; Hsu, F.C.; Ko, S.S. Arabidopsis Hsa32, a novel heat shock protein, is essential for acquired thermotolerance during long recovery after acclimation. Plant Physiol. 2006, 140, 1297-1305.

8. Shakeel, S.N.; Heckathorn, S.; Luthe, D.S. Analysis of gene sequences indicates that quantity not quality of chloroplast small HSPs improves thermotolerance in C4 and CAM plants. Plant Cell Rep. 2012, 31, 1943-1957.

9. Khurana, N.; Chauhan, H.; Khurana, P. Wheat chloroplast targeted sHSP26 promoter confers heat and abiotic stress inducible expression in transgenic Arabidopsis plants. Plos One. 2013, 8 , e54418.

10. Zou, J.; Liu, C.F.; Liu, A.L.; Zou, D.; Chen, X.B. Overexpression of OsHsp17.0 and OsHsp23.7 enhances drought and salt tolerance in rice. J Plant Physiol. 2012, 169, 628-635.

11. Kaur, H.; Petla, B.P.; Kamble, N.U.; Singh, A.; Rao, V.; Salvi, P.; Ghosh, S.; Majee, M. Differentially expressed seed aging responsive heat shock protein OsHSP18.2 implicates in 
373

374

375

376

377

378

379

380

381

382

383

384

385

386

387

388

389

390

391

392

393

394

395

396

397

398

399

400

401

402

403

404

405

406

407

408

409

410

411

412

413

414

415

416

417

418

seed vigor, longevity and improves germination and seedling establishment under abiotic stress. Front Plant Sci. 2015, 6, 713.

12. Chauhan, H.; Khurana, N.; Nijhavan, A.; Khurana, J.P.; Khurana, P. The wheat chloroplastic small heat shock protein (sHSP26) is involved in seed maturation and germination and imparts tolerance to heat stress. Plant Cell Environ. 2012, 35, 1912-1931.

13. Wang, J.; Gao, X.; Dong, J.; Tian, X.; Wang, J.; Palta, J.A.; Xu, S.; Fang, Y.; Wang, Z. OverExpression of the Heat-Responsive Wheat Gene TaHSP23.9 in Transgenic Arabidopsis Conferred Tolerance to Heat and Salt Stress. Plant Sci. 2020, 11, 243.

14. Mu, C.J.; Zhang, S.J.; Yu, G.Z.; Chen, N.; Li, X.F.; Liu, H. Overexpression of small heat shock protein LimHSP16.45 in Arabidopsis enhances tolerance to abiotic stresses. Plos One. 2013, 8, e82264.

15. Li, M.; Ji, L.; Jia, Z.; Yang, X.; Meng, Q.; Guo, S. Constitutive expression of CaHsp22.5 enhances chilling tolerance in transgenic tobacco by promoting the activity of antioxidative enzymes. Funct Plant Biol. 2018, 45, 575-585.

16. Sun, X.; Sun, C.; Li, Z.; Hu, Q.; Han, L.; Luo, H. AsHSP17, a creeping bentgrass small heat shock protein modulates plant photosynthesis and ABA-dependent and independent signalling to attenuate plant response to abiotic stress. Plant Cell Environ. 2016, 39, 1320-1337.

17. Sun, X.; Zhu, J.; Li, X.; Li, Z.; Han, L.; Luo, H. AsHSP26.8a, a creeping bentgrass small heat shock protein integrates different signaling pathways to modulate plant abiotic stress response. BMC Plant Biol. 2020, 20, 184.

18. Guo, S.; Zhang, J.; Sun, H.; Salse, J.; Lucas, W.J.; Zhang, H.; Zheng, Y.; Mao, L.; Ren, Y.; Wang, Z.; Min, J.; Guo, X.; Murat, F.; Ham, B.K.; Zhang, Z.; Gao, S.; Huang, M.; Xu, Y.; Zhong, S.; Bombarely, A.; Mueller, L.A.; Zhao, H.; He, H.; Zhang, Y.; Zhang, Z.; Huang, S.; Tan, T.; Pang, E.; Lin, K.; Hu, Q.; Kuang, H.; Ni, P.; Wang, B.; Liu, J.; Kou, Q.; Hou, W.; Zou, X.; Jiang, J.; Gong, G.; Klee, K.; Schoof, H.; Huang, Y.; Hu, X.; Dong, S.; Liang, D.; Wang, J.; Wu, K.; Xia, Y.; Zhao, X.; Zheng, Z.; Xing, M.; Liang, X.; Huang, B.; Lv, T.; Wang, J.; Yin, Y.; Yi, H.; Li, R.; Wu, M.; Levi, A.; Zhang, X.; Giovannoni, J.J.; Wang, J.; Li, Y.; Fei, Z.; Xu, Y. The draft genome of watermelon (Citrullus lanatus) and resequencing of 20 diverse accessions. Nat Genet. 2013, 45, 51-58.

19. Yetr, H.; Uygur, V. Plant Growth and Mineral Element Content of Different Gourd Species and Watermelon under Salinity Stress. TURK J AGRIC FOR. 2009, 33, 65-77.

20. He, Y.; Fan, M.; Sun, Y.; Li, L. Genome-Wide Analysis of Watermelon HSP20s and Their Expression Profiles and Subcellular Locations under Stresses. Int J Mol Sci. 2019, 20, 12.

21. Tamura, K.; Peterson, D.; Peterson, N.; Stecher, G.; Nei, M.; Kumar, S. MEGA5: Molecular evolutionary genetics analysis using maximum likelihood, evolutionary distance, and maximum parsimony methods. Mol Biol Evol. 2011, 28, 2731-2739.

22. Thompson, J.D.; Gibson, T.J.; Plewniak, F.; Jeanmougin, F.; Higgins, D.G. The CLUSTAL_X windows interface: Flexible strategies for multiple sequence alignment aided by quality analysis tools. Nucleic Acids Res. 1997, 25, 4876-4882.

23. Xiuren, Z.; Rossana, H.; Shih-Shun, L.; Qi-Wen, N.; Nam-Hai, C. Agrobacterium-mediated transformation of Arabidopsis thaliana using the floral dip method. Nat Protoc. 2006, 2, 1-6.

24. Abbasi, H.; Jamil, M.; Haq, A.; Ali, S.; Ahmad, R.; Malik, Z.; Parveen. Salt stress manifestation on plants, mechanism of salt tolerance and potassium role in alleviating it: A review. ZEMDIRBYSTE. 2016, 103, 229-238.

25. Guo, L.M.; Li, J.; He, J.; Liu, H.; Zhang, H.M. A class I cytosolic HSP20 of rice enhances heat and salt tolerance in different organisms. SCI REP-UK. 2020, 10, 1383. 
419

420

421

422

423

424

425

426

427

428

429

430

431

432

433

434

435

436

437

438

439

440

441

442

443

444

445

446

447

448

449

450

451

452

26. Kim, D.H.; Xu, Z.Y.; Na, Y.J.; Yoo, Y.J.; Lee, J.; Sohn, E.J.; Hwang, I. Small Heat Shock Protein Hsp17.8 Functions as an AKR2A Cofactor in the Targeting of Chloroplast Outer Membrane Proteins in Arabidopsis. Plant Physiol. 2011, 157, 132-146.

27. Francino, M.P. An adaptive radiation model for the origin of new gene functions. Nat Genet. 2005, 37, 573-578.

28. Banerjee, A.; Roychoudhury, A. Abscisic-acid-dependent basic leucine zipper (bZIP) transcription factors in plant abiotic stress. Protoplasma. 2017, 254, 3-16.

29. Fujita, Y.; Fujita, M.; Shinozaki, K.; Yamaguchi-Shinozaki, K. ABA mediated transcriptional regulation in response to osmotic stress in plants. J Plant Res. 2011, 124, 509-525.

30. Roychoudhury, A.; Paul, S.; Basu, S. Cross-talk between abscisic acid dependent and abscisic acid-independent pathways during abiotic stress. Plant Cell Rep. 2013, 32, 985-1006.

31. Yoshida, T.; Mogami, J.; Yamaguchi-Shinozaki, K. ABA-dependent and ABA-independent signaling in response to osmotic stress in plants. Curr Opin in Plant Biol. 2014, 21, 133-139.

32. Ciftci-Yilmaz, S.; Morsy, M.R.; Song, L.; Coutu, A.; Krizek, B.A.; Lewis, M.W.; Warren, D.; Cushman, J.; Connolly, E.L.; Mittler, R. The EAR-motif of the Cys2/His2-type zinc finger protein Zat7 plays a key role in the defense response of Arabidopsis to salinity stress. $J$ Biol Chem. 2007, 282, 9260-9268.

33. Nguyen, N.H.; Cheong, J. H2A.Z-containing nucleosomes are evicted to activate AtMYB44 transcription in response to salt stress. Biochem Bioph Res Co. 2018, 499, 1039-1043.

34. Yu, Y.; Ni, Z.; Chen, Q.; Qu, Y. The wheat salinity-induced R2R3-MYB transcription factor TaSIM confers salt stress tolerance in Arabidopsis thaliana. Biochem Biophys Res Commun. 2017, 491, 642-648.

35. Wei, Q.; Luo, Q.; Wang, R.; Zhang, F.; He, Y.; Zhang, Y.; Qiu, D.; Li, K.; Chang, J.; Yang, G.; He, G. A wheat R2R3-type MYB transcription factor TaODORANT1 positively regulates drought and salt stress responses in transgenic tobacco plants. Front Plant Sci. 2017, 8, 1374.

36. Seo, J.S.; Sohn, H.B.; Noh, K.; Jung, C.; An, J.H.; M. Donovan, C.; Somers, D.A.; Kim, D.I.; Jeong, S.C.; Kim, C.G.; Kim, H.M.; Lee, S.H.; Choi, Y.D.; Moon, T.W.; Kim, C.H.; Cheong, J.J. Expression of the Arabidopsis AtMYB44 gene confers drought/salt-stress tolerance in transgenic soybean. Mol Breeding. 2012, 29, 601-608.

37. Persak, H.; Pitzschke, A. Dominant repression by Arabidopsis transcription factor MYB44 causes oxidative damage and hypersensitivity to abiotic stress. Int J Mol Sci. 2014, 15, 25172537.

Peerj reviewing PDF | (2020:07:50605:1:1:NEW 28 Oct 2020) 
Figure 1

Figure 1 Phylogenetic and amino acid sequence analysis of CIHSP22.8 orthologs in various species.

Figure 1 Phylogenetic and amino acid sequence analysis of CIHSP22.8 orthologs in various species. (A) Amino acid sequence alignment of $\alpha$-crystallin ACD/HSP20 domain from Arabidopsis (At), rice (Os), tomato (SI), soybean (Gm), switchgrass (Pv), cucumber (Cs), and watermelon $(\mathrm{Cl})$. Conserved region I and II were indicated by red boxes. (B) Phylogenetic analysis of ClHSP22.8 protein orthologs from these orthologs. Phylogenetic analysis based on full-length protein sequences was performed using the MEGA 5.0 program by the neighborjoining (NJ) method with 1000 bootstrap replicates.

A
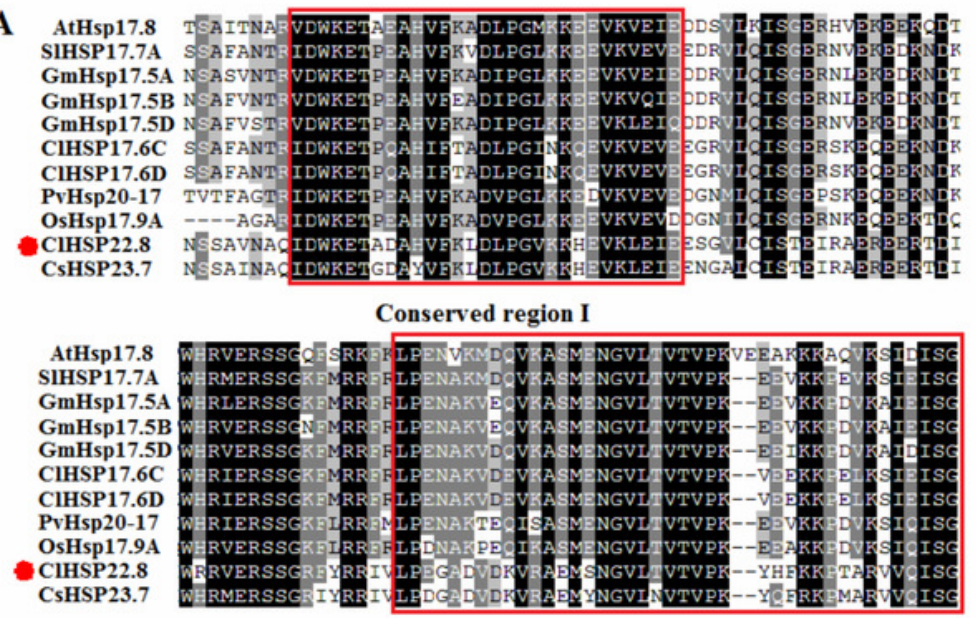

Conserved region II
B

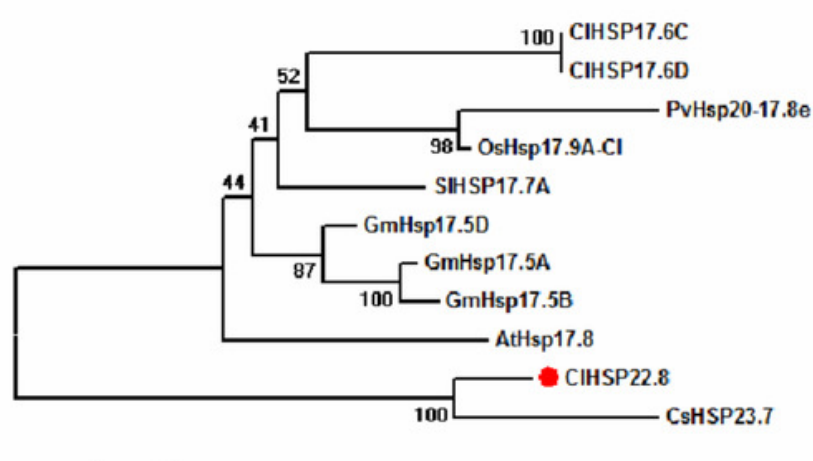




\section{Figure 2}

Figure 2 Spatial and temporal expression patterns of CIHSP22.8.

Figure 2 Spatial and temporal expression patterns of CIHSP22.8. (A) CIHSP22.8 expression levels in root (R), stem (S), leaf (L), female flower (Ff), male flower (Mf), and fruit ( $\mathrm{Fr}$ ) of watermelon via quantitative real-time PCR analysis (qRT-PCR). Histochemical GUS assays of ProCIHSP22.8::GUS in Arabidopsis. GUS protein was expressed in 7-day-old seedlings with GUS-PBI101 (mock) (B) and ProCIHSP22.8:: GUS (C). (D - H) represents the GUS signal was expressed in root $(D)$, stem $(E)$, leaf $(F)$, flower $(G)$, and silique $(H)$ of adult Arabidopsis plants.
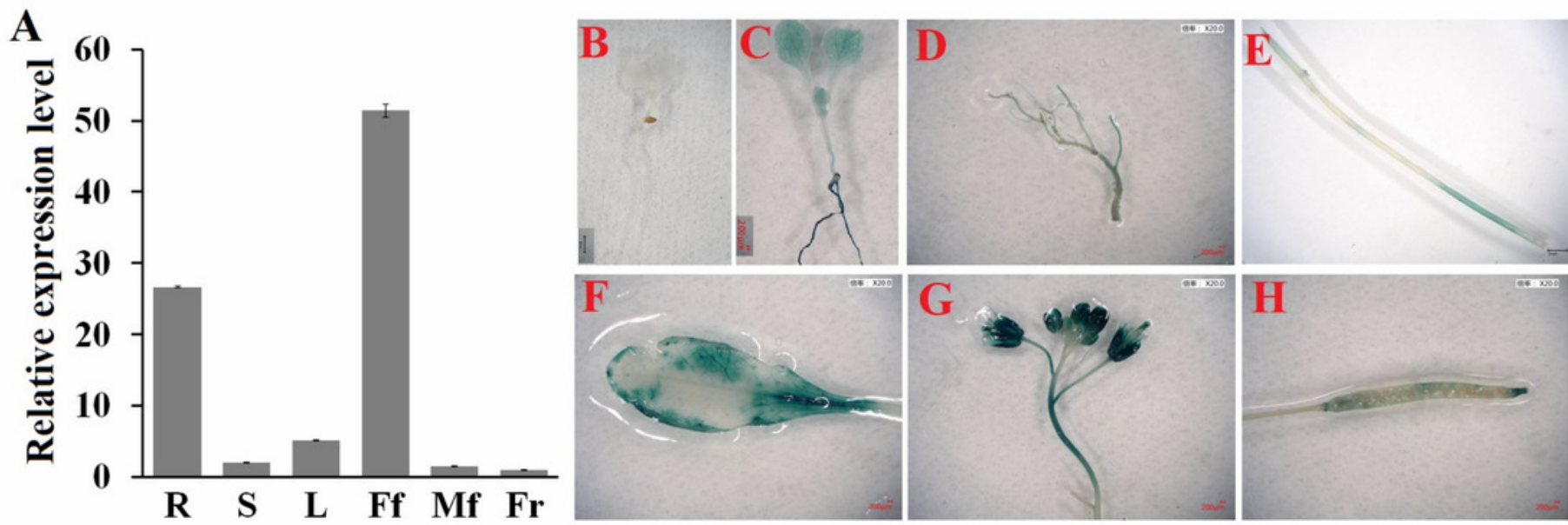


\section{Figure 3}

Figure 3 Expression profiles of CIHSP22.8 in response to abscisic acid (ABA) and salt stress.

Figure 3 Expression profiles of CIHSP22.8 in response to abscisic acid (ABA) and salt stress. CIHSP22.8 expression levels in watermelon leaves exposed to $100 \mu \mathrm{M}$ ABA (A) and $200 \mathrm{mM}$ $\mathrm{NaCl}(\mathrm{B})$ at $0,1,4$, and $12 \mathrm{~h}$ via qRT-PCR. The asterisks on the top of the columns indicate significant differences from the value at $0 \mathrm{~h} . * \mathrm{P}<0.05$; $* \mathrm{P}<0.01$ (C) Cis-elements analysis of CIHSP22.8 promoter sequence. (D) Histochemical GUS assays of 7-day-old transgenic seedlings with empty vector. (E-F) Histochemical GUS assays of 7-day-old transgenic seedlings with ProCIHSP22.8::GUS exposed to ABA and $\mathrm{NaCl}$ for $24 \mathrm{~h}$. 

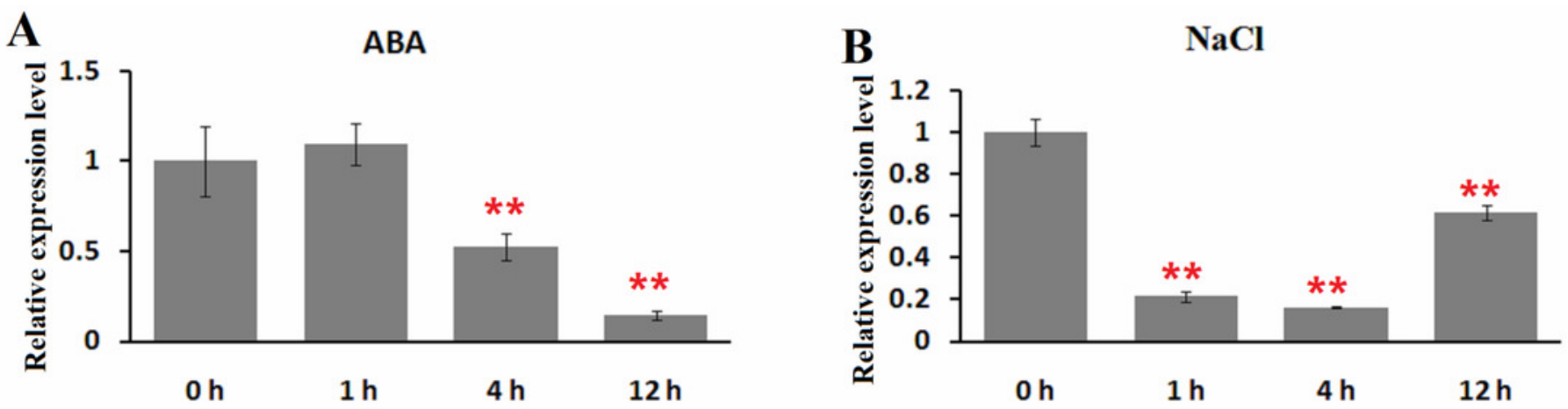

C

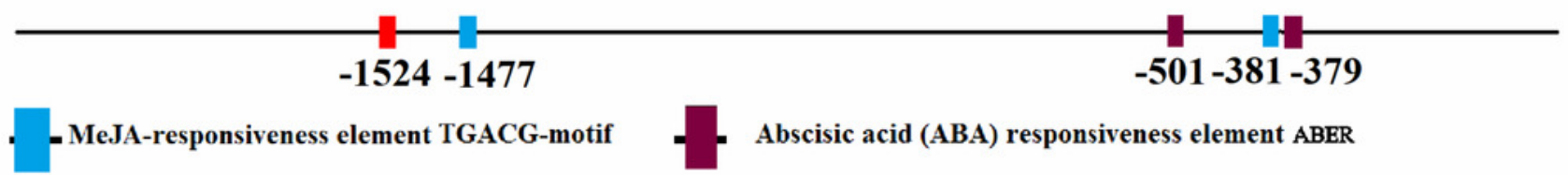

Anaerobic responsiveness element ARE

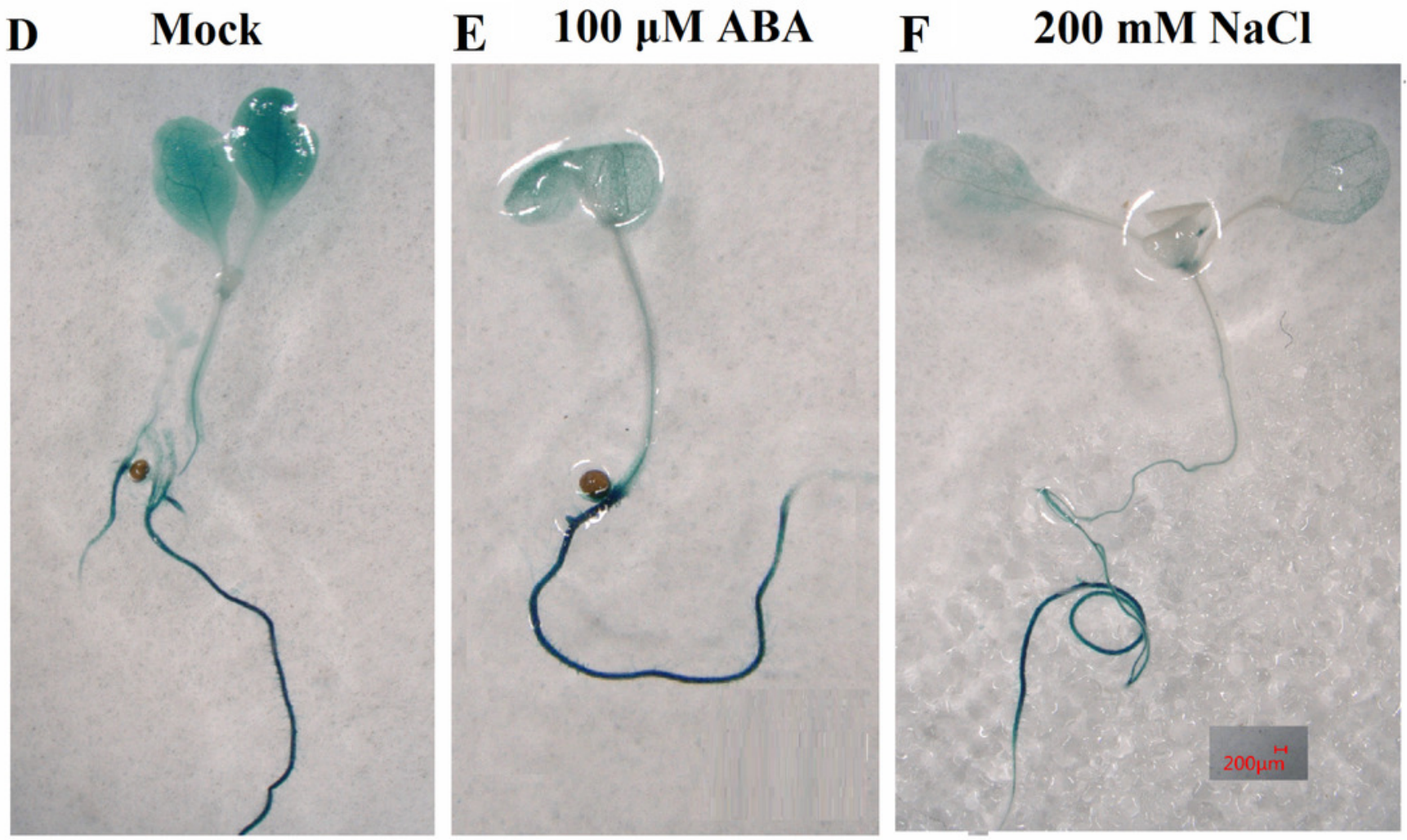




\section{Figure 4}

Figure 4 Seedling assay of CIHSP22.8-overexpressing lines and wild type (WT) in response to $\mathrm{ABA}$ and $\mathrm{NaCl}$ treatment.

Figure 4 Seedling assay of CIHSP22.8-overexpressing lines and wild type (WT) in response to $\mathrm{ABA}$ and $\mathrm{NaCl}$ treatment. (A) Relative gene expression of CIHSP22.8 in different overexpression Arabidopsis transgenic lines OE22.8-1 to OE22.8-4. (B) The root length of the OE22.8 transgenic and WT plants in the presence of $100 \mathrm{mM} \mathrm{ABA}$ and $200 \mathrm{mM} \mathrm{NaCl}$ for 7 days after germination, respectively. Growth of the transgenic and WT plants under normal condition (C) and in the presence of $100 \mathrm{mM} \mathrm{ABA}(D)$ and $200 \mathrm{mM} \mathrm{NaCl}(E)$ for 7 days after germination. * and ** represent significant differences between WT and OE22.8 lines at values of $P<0.05$ and $P<0.01$, respectively, as determined by Student's $t$-test.

A

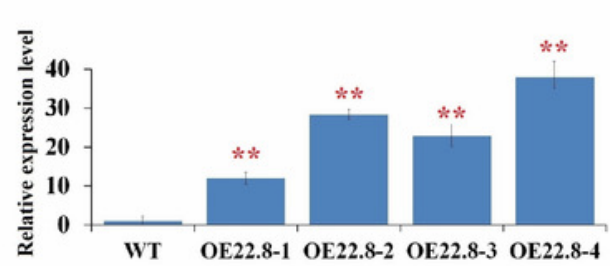

B

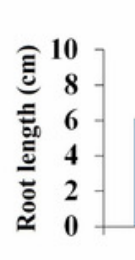

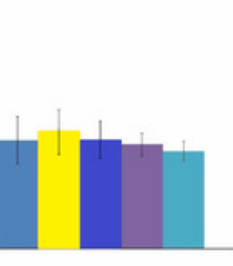

Mock

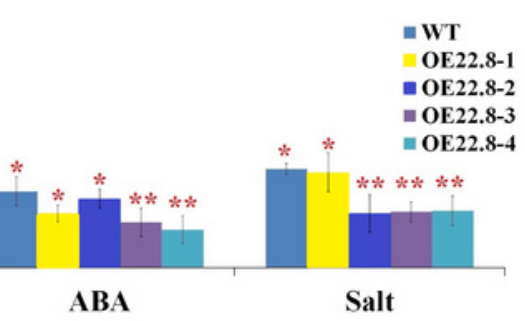

$\begin{array}{lllll}C & \text { WT } & \text { OE22.8-1 } & \text { OE22.8-2 } & \text { OE.22.8-3 OE22.8-4 }\end{array}$

D wT

$\begin{array}{llllll}\text { OE22.8-1 } & \text { OE22.8-2 } & \text { OE22.8-3 } & \text { OE22.8-4 } & \text { E } & \text { WT }\end{array}$

OE22.8-1 OE22.8-2 OE22.8-3 OE22.8-4
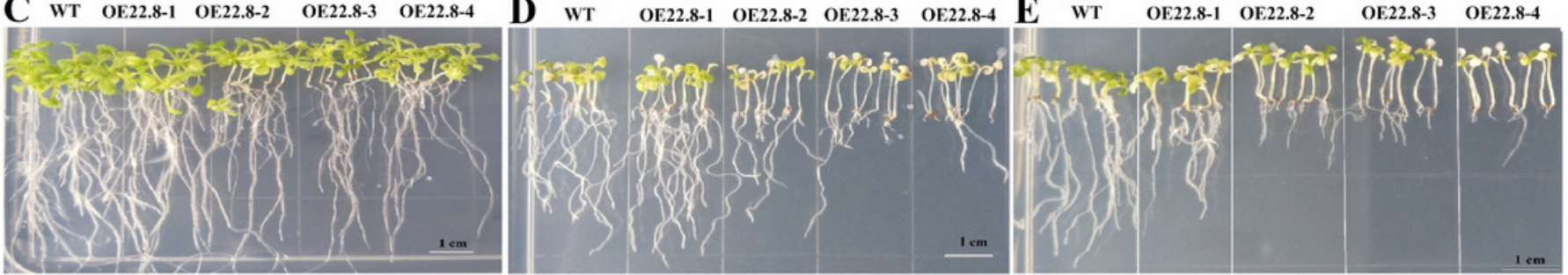


\section{Figure 5}

Figure 5 Expression profiles of ABA- and stress-related genes in WT and CIHSP22.8 overexpressing Arabidopsis plants.

Figure 5 Expression profiles of ABA- and stress-related genes in WT and CIHSP22.8 overexpressing Arabidopsis plants. * and ** represent significant differences from the control at values of $P<0.05$ and $P<0.01$, respectively, as determined by Student's $t$-test.
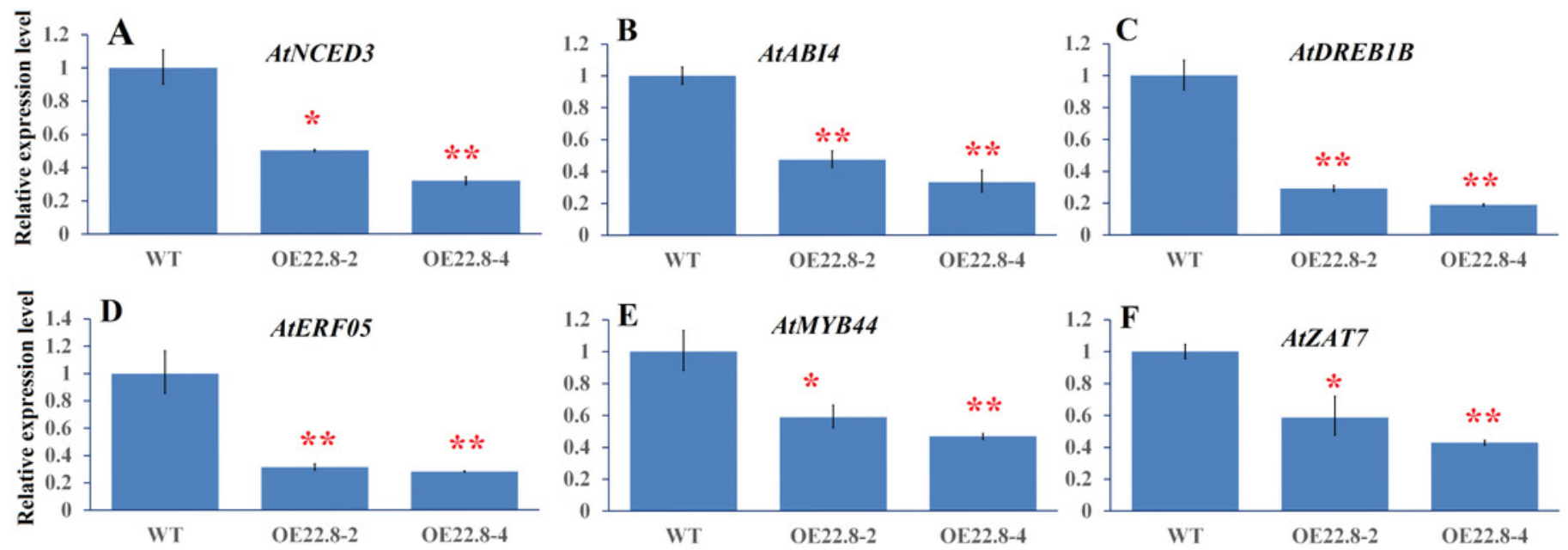


\section{Figure 6}

Figure 6 Expression profiles of ABA- and stress-related genes in three-week-old WT and CIHSP22.8 overexpressing Arabidopsis plants $24 \mathrm{~h}$ after salt treatment.

Figure 6 Expression profiles of ABA- and stress-related genes in three-week-old WT and CIHSP22.8 overexpressing Arabidopsis plants $24 \mathrm{~h}$ after salt treatment. The changes observed in WT were normalized. * and ** represent significant differences between the mock- and salt-treatment OE22.8 plants at values of $P<0.05$ and $P<0.01$, respectively, as determined by Student's $t$-test.
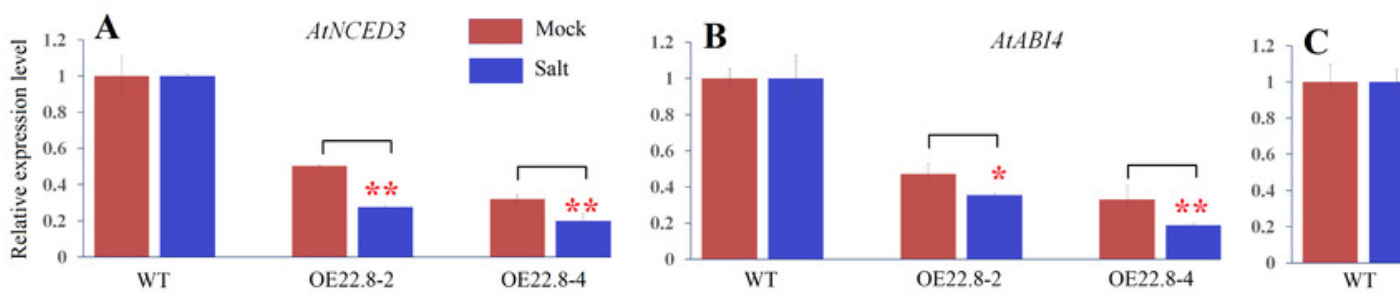

$A t D R E B I B$
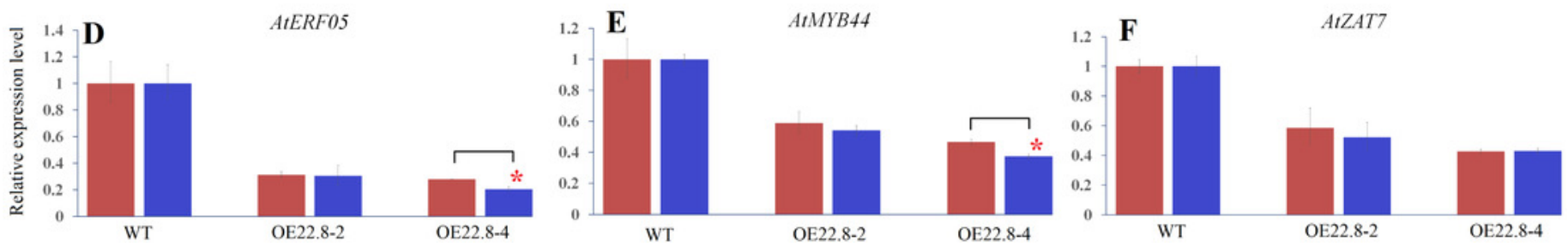

AtZAT7 


\section{Figure 7}

Figure 7 A proposed model for the roles of CIHSP22.8 in salt stress resistance in Arabidopsis.

Figure 7 A proposed model for the roles of CIHSP22.8 in salt stress resistance in Arabidopsis. CIHSP22.8 is involved in ABA biosynthesis, ABA-dependent and independent, and other stress responsive signaling pathways to modulate plant response to salt stress. Abbreviations: AtNCED3, Arabidopsis 9-cis epoxycarotenoid dioxygenase 3; AtABI4, Arabidopsis ABA insensitive 4; AtERF05, ethylene response factor 05; AtDREB1B, Arabidopsis dehydrationresponsive element-binding protein 1B; AtMYB44, Arabidopsis myb domain protein 44 . Arrows indicate positive regulation, whereas lines ending with a bar indicate negative regulation. 


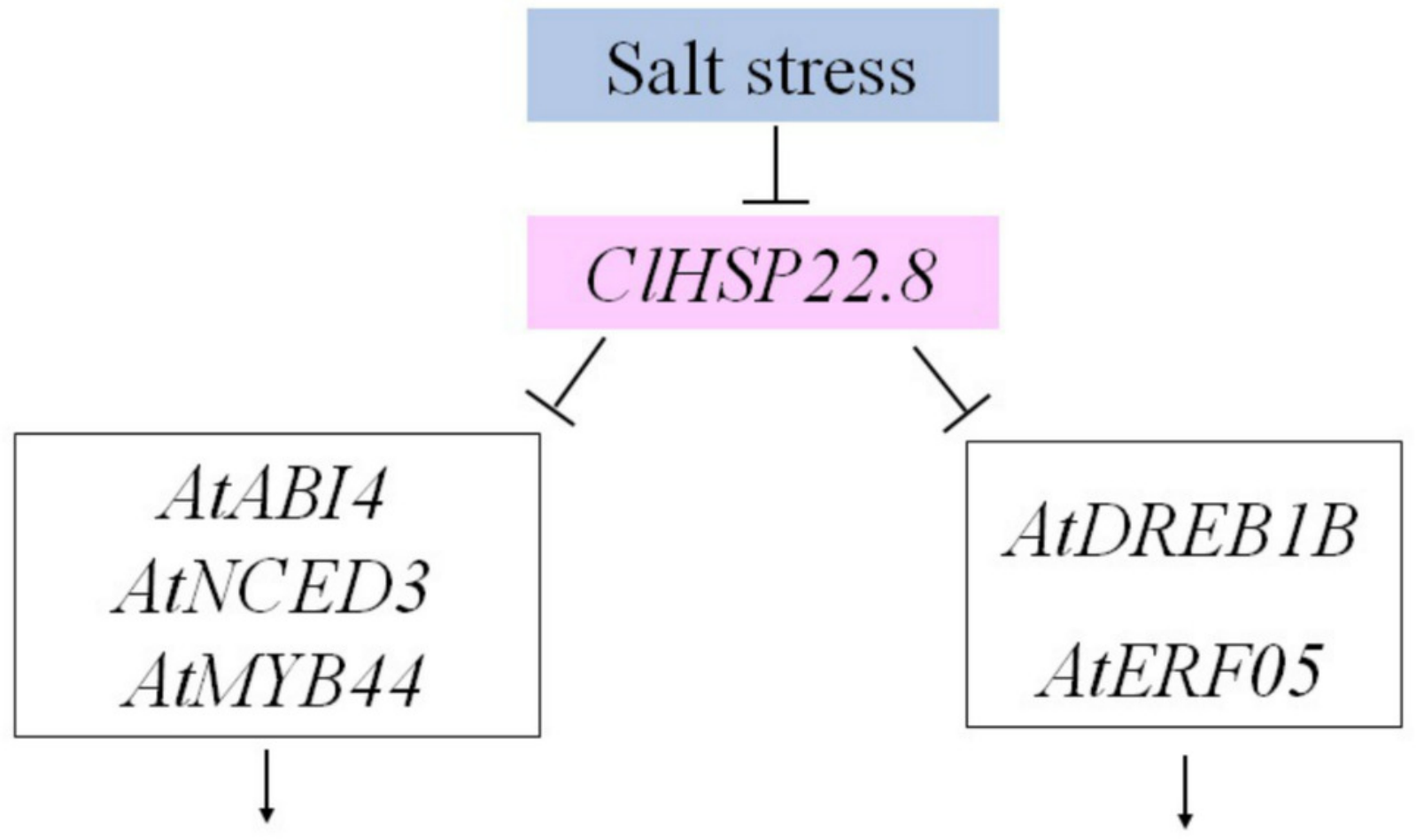

ABA biosynthesis and responsive genes $\downarrow$

ABA-dependent signalling pathway
Stress responsive genes

ABA-independent
signalling pathway

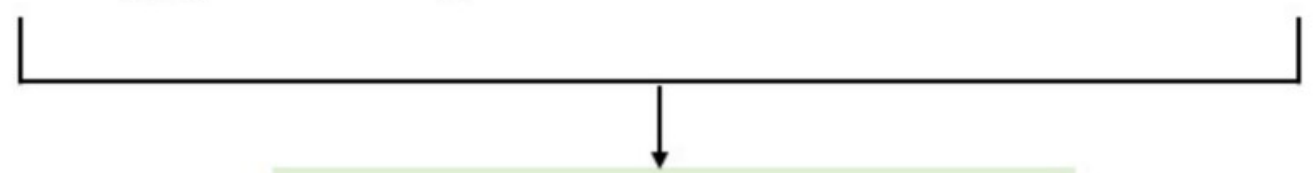

Salt stress resistance 